\title{
Metabolic Syndrome and Hypertension Subtypes among Untreated Hypertensive Rural Chinese
}

Xingang Zhang1, Shuang $\mathrm{Yao}^{2}$, Zhaoqing Sun², Liqiang Zheng ${ }^{2}$, Changlu $\mathrm{Xu}^{2}$, Jue $\mathrm{Li}^{3}$, Dayi Hu${ }^{3}$ and $\mathrm{Yingxian} \mathrm{Sun}^{1 *}$

${ }^{1}$ Department of Cardiology, The First Affiliated Hospital of China Medical University, Shenyang, P.R. China

${ }^{2}$ Department of Cardiology, Shengjing Hospital of China Medical University, Shenyang, P.R. China

${ }^{3}$ Heart, Lung and Blood Vessel Center, Tongji University, Shanghai, P.R. China

\begin{abstract}
Background: High blood pressure is a major contributor to metabolic syndrome (MS) and the selection for hypertension in most cases is associated with higher prevalence of MS. However, the relation between hypertension subtypes and MS is not well defined.

Methods: A cross-sectional survey was conducted during 2004-2006, which undertook cluster multistage sampling to a representation sample in rural villages of Liaoning, China. A total of 4273 untreated hypertensive subjects aged $\geq 35$ years were selected. The metabolic syndrome was defined by the National Cholesterol Education Program. Hypertension consisted of three subtypes: Isolated Systolic Hypertension (ISH), Isolated Diastolic Hypertension (IDH), and Systolic-Diastolic Hypertension (SDH).

Results: Of the study population $23.4 \%$ (men: $12.0 \%$, women: $33.4 \%$ ) had metabolic syndrome. Overall, $24.4 \%$ (men: $22.2 \%$, women: $26.3 \%$ ), $10.0 \%$ (men: 9.5 , women: $10.5 \%$ ), and $65.6 \%$ (men: $68.4 \%$, women: $63.3 \%$ ) of the untreated hypertensive individuals had ISH, IDH, and SDH, respectively. The MS prevalence in untreated persons was 23.2\% (men: $11.3 \%$, women: $31.9 \%$ ) for ISH, $18.7 \%$ (men: $9.5 \%$, women: $26.1 \%$ ) for IDH, and $24.1 \%$ (men: $12.5 \%$, women: $35.2 \%$ ) for SDH. Among those with MS, $24.1 \%$ (men: $20.9 \%$, women: $25.2 \%$ ) had ISH, $8.0 \%$ (men: $7.5 \%$, women: $8.2 \%$ ) had IDH, and $67.8 \%$ (men: $71.5 \%$, women: $66.7 \%$ ) had SDH.
\end{abstract}

Conclusions: SDH was the most prevalent hypertensive subtype; however, the prevalence of MS was similar in persons with ISH or SDH. Additionally, the high frequency of SDH in the hypertensive population made SDH the most common hypertensive subtype in persons with MS.

Keywords: Metabolic syndrome; Hypertension; Rural population; Epidemiology

\section{Introduction}

Metabolic syndrome (MS), a constellation of risk factors associated with vascular diseases is becoming a major public health issue not only prevalent among US and European populations [1,2], but also among the developing countries. Several prospective studies have shown that the MS was associated with increased morbidity or mortality not only for patients with cardiovascular disease $[3,4]$ but also for patients with stroke events $[5,6]$. As an element of MS, hypertension is the key role associated with the significantly increasing cardiovascular mortality [7]. Studies have also indicated that hypertension subtypes were significantly associated with cardiac and cerebrovascular events. Previous evidence from prospective epidemiological studies reported that isolated systolic hypertension (ISH) was the single greatest risk factor, other than age, for the development of cardiovascular diseases in the elderly population [8,9]. Given that individuals with ISH had higher prevalence of left ventricular hypertrophy and carotid atherosclerosis than subjects with diastolic hypertension despite lower mean blood pressure [10]. Other studies showed that systolic-diastolic hypertension $(\mathrm{SDH})$ patients were at the highest risk of cardiovascular disease or stroke among all the hypertensives [11,12]. However the comparative relation of hypertension subtypes to the odds of having MS has not been well defined. The goal of the current study was therefore to estimate whether each hypertension subtype were related to the likelihood of MS

\section{Methods}

\section{Study population}

The procedures followed were in accordance with ethical standards of the responsible committee on human experimentation of China
Medical University. A cross-sectional survey was conducted from Oct 2004 to June 2006 in Liaoning province of China. The study used a cluster multistage sampling method, which included samples from rural hypetensives in the northern, southern, western, eastern, and the central regions of Fuxin county in Liaoning province. Only 1 small town was selected from each region. Finally, 10 rural villages near each small town were randomly selected from different geographic areas. In total, 5 small towns from these regions, and 50 rural villages were selected to a resident group. A total of 4273 untreated hypertensive subjects (men 1998, women 2275) aged $\geq 35$ years were selected.

\section{Measurements}

The baseline surveys were conducted by local doctors with home visits method. During the interview and examination, doctors administered a standard questionnaire including questions related to demographic variables (age, sex, and race). Blood pressure (BP) was measured with a checked electronic sphygmomanometer (Omron) and three blood pressure measurements were taken. At the study site, anthropometric measurements including waist circumference, height, and weight were obtained from each participant with standard

*Corresponding author: Yingxian Sun, M.D. Ph.D, Department of cardiology, The First Affiliated Hospital of China Medical University, Heping District, Nanjing North Street, No. 155, Shenyang, 110001, P.R. China, Tel: 86-24-83282688; Fax: 86-2483282688; E-mail: professor_sun@yahoo.cn

Received March 06, 2012; Accepted March 29, 2012; Published March 31, 2012

Citation: Zhang X, Yao S, Sun Z, Zheng L, Xu C, et al. (2012) Metabolic Syndrome and Hypertension Subtypes among Untreated Hypertensive Rural Chinese. J Nutr Disorders Ther 2:111. doi:10.4172/2161-0509.1000111

Copyright: $\odot 2012$ Zhang X, et al. This is an open-access article distributed under the terms of the Creative Commons Attribution License, which permits unrestricted use, distribution, and reproduction in any medium, provided the original author and source are credited. 
protocols. Subjects were asked to fast for at least 12 hours before blood collection. Blood samples were obtained from an antecubital vein into vacutainer tubes containing EDTA. Blood chemical analyses were performed at a central, certified laboratory. Blood lipids and fasting glucose were analyzed enzymatically on an Olympus AU640 autoanalyzer.

\section{Definitions}

MS is defined according to ATP III criteria [13] as the presence of three or more of the following components:

1) Elevated BP: systolic BP $\geq 130 \mathrm{mmHg}$, diastolic BP $\geq 85$ $\mathrm{mmHg}$, or use of antihypertensive medications; 2) Elevated plasma glucose: fasting glucose $\geq 6.1 \mathrm{mmol} / \mathrm{L} \quad(110 \mathrm{mg} / \mathrm{dl})$, self-reported physician diagnosed diabetes, or self-reported use of hypoglycemic agent or insulin; 3) Elevated serum TG: $\geq 1.7 \mathrm{mmol} / \mathrm{L}$ (150mg/dl); 4) Decreased HDL-cholesterol: $<1.0 \mathrm{mmol} / \mathrm{L}(40 \mathrm{mg} / \mathrm{dL})$ for men and $<1.3 \mathrm{mmol} / \mathrm{L}(50 \mathrm{mg} / \mathrm{dl})$ for women; and 5) Higher WC: $\geq 102 \mathrm{~cm}$ for men and $\geq 88 \mathrm{~cm}$ for women. Untreated hypertension was considered without antihypertensive treatment and present if any of the following conditions were met: systolic blood pressure (SBP) $\geq 140 \mathrm{mmHg}$, diastolic blood pressure (DBP) $\geq 90 \mathrm{mmHg}$. Subtypes of hypertension were defined according to the baseline SBP, DBP: ISH (SBP $\geq 140$ $\mathrm{mmHg}$ and $\mathrm{DBP}<90 \mathrm{mmHg}$ ); $\mathrm{SDH}(\mathrm{SBP} \geq 140 \mathrm{mmHg}$ and $\mathrm{DBP} \geq 90$ $\mathrm{mmHg}$ ); isolated diastolic hypertension (IDH) $(\mathrm{SBP}<140 \mathrm{mmHg}$ and $\mathrm{DBP} \geq 90 \mathrm{mmHg}$ ).

\section{Statistical analysis}

To account for the clustering and stratification of the survey design, and to adjust for non-response, the data were weighted to match the age distribution of the 2000 China Population Census data aged $\geq 35$ years, unless otherwise stated. The weighting factor was based on the probability of selection in each cluster. Therefore, all prevalence relate to the total 2000 China population aged $\geq 35$ years. All data analyses were conducted by use of SPSS 11.5 (SPSS Inc., Chicago, IL) statistical software package. Continuous variables were presented as mean values and standard deviation. Categorical variables were presented as frequencies. The prevalence estimates of MS categories were calculated by age, gender and hypertension subtypes -specific proportions. A chisquare test was used to examine gender and hypertension subtypes differences. For all comparisons, $p$-values $<0.05$ were considered statistically significant.

\section{Results}

\section{Basic characteristics of study population}

The characteristics of the untreated hypertensive individuals enrolled in this study, as stratified by gender, are shown in Table 1. All subjects were selected from 35 to 92 years old and the average ages of the men and the women were $57.41 \pm 11.57$ and $55.70 \pm 11.20$ years, respectively. Compare with women, untreated hypertensive men had significantly higher prevalence of SDH, levels of age and DBP. However, levels of HDL-cholesterol, prevalence of ISH were lower in untreated hypertensive women. The SBP levels, waist circumference levels, triglycerid levels and prevalence of IDH were not shown significant differences across different gender.

\section{Prevalence of metabolic syndrome and hypertension subtypes}

As shown in Table 2, approximately $23.4 \%$ of the untreated hypertensive individuals had MS. The prevalence of MS was significantly higher in women than that in men $(p<0.001$.). For men, the prevalence of MS declined gradually with age. For women, the highest prevalence of MS was in the decade aged 55-64; however, it decreased obviously when age $\geq 65$ years.

Overall, 24.4\% (men: 22.2\%, women: 26.3\%), 10.0\% (men: 9.5, women: $10.5 \%$ ), and $65.6 \%$ (men: $68.4 \%$, women: $63.3 \%$ ) of the untreated hypertensive individuals had ISH, IDH, and SDH, respectively. The MS prevalence in untreated persons was $23.2 \%$ (men: $11.3 \%$, women: $31.9 \%$ ) for ISH, $18.7 \%$ (men: $9.5 \%$, women: $26.1 \%$ ) for IDH, and $24.1 \%$ (men: $12.5 \%$, women: $35.2 \%)$ for SDH $(p<0.05)$. Compared with men, untreated hypertensive women had significantly higher prevalence of MS among different hypertensive subtypes ( $p<$ 0.001) (Figure 1).

As presents in Figure 2 and 3, among those with MS, 24.1\% (men: 20.9\%, women: $25.2 \%$ ) had ISH, $8.0 \%$ (men: $7.5 \%$, women: $8.2 \%$ ) had IDH, and 67.8\% (men: 71.5\%, women: 66.7\%) had SDH; for those without MS the corresponding figure was $24.4 \%, 10.6 \%$, and $65.0 \%$, respectively. In general, for people with MS, the proportion of individuals with IDH and SDH was progressively lower and the proportion of individuals with ISH was progressively higher with increments of age when age $\geq 45$ years, whereas this was happen when age $\geq 35$ years in people without MS.

\section{Prevalence of MS components clusters by hypertension subtypes}

The prevalence of MS components clusters as follow: for ISH, $34.5 \%, 17.8 \%, 4.7 \%$, and $0.7 \%$ of the study individuals had $1,2,3$, and 4 components, respectively; for IDH the corresponding figure was $38.2 \%, 13.8 \%, 4.2 \%$, and $0.7 \%$, respectively; and for SDH $36.4 \%, 18.8 \%$, $4.6 \%, 0.8 \%$.

\section{Discussion}

The main findings of this study were that the highest prevalence of hypertension subtypes was SDH (65.6\%) among untreated rural hypertensive Chinese population; subjects with $\mathrm{SDH}$ had the highest prevalence of MS (24.1\%) as compared with those individuals with

\begin{tabular}{lccc}
\hline Characteristic & Men $(\mathrm{n}=1998)$ & Women $(\mathrm{n}=2275)$ & $p$ values \\
\hline Age (years) & $57.41 \pm 11.57$ & $55.70 \pm 11.20$ & $<0.001$ \\
Systolic blood pressure $(\mathrm{mmHg})$ & $159.31 \pm 22.25$ & $158.84 \pm 21.13$ & 0.462 \\
Diastolic blood pressure $(\mathrm{mmHg})$ & $95.70 \pm 12.46$ & $93.87 \pm 11.33$ & $<0.001$ \\
Waist circumference $(\mathrm{cm})$ & $82.69 \pm 9.00$ & $82.35 \pm 10.23$ & 0.258 \\
HDL cholesterol $(\mathrm{mmol} / \mathrm{L})$ & $1.43 \pm 0.33$ & $1.45 \pm 0.33$ & 0.016 \\
Triglycerid $(\mathrm{mmol} / \mathrm{L})$ & $1.69 \pm 1.90$ & $1.76 \pm 1.38$ & 0.174 \\
Fasting blood glucose $(\mathrm{mmol} / \mathrm{L})$ & $5.70 \pm 1.50$ & $5.71 \pm 1.83$ & 0.806 \\
Isolated systolic hypertension $(\%)$ & 22.2 & 26.3 & 0.002 \\
Isolated diastolic hypertension (\%) & 9.5 & 10.5 & 0.276 \\
Systolic-diastolic hypertension (\%) & 68.4 & 63.3 & $<0.001$
\end{tabular}

Table 1: Clinical characteristics of the untreated individuals.

\begin{tabular}{llll}
\hline Age $($ years $)$ & Men $(n=1998)$ & Women $(n=2275)$ & All $(n=4273)$ \\
\hline $35-44$ & $44(13.8)$ & $138(32.4)$ & $182(24.5)$ \\
$45-54$ & $71(13.7)$ & $217(31.8)$ & $288(24.0)$ \\
$55-64$ & $72(12.1)$ & $259(39.0)$ & $331(26.2)$ \\
$\geq 65$ & $52(9.2)$ & $145(28.9)$ & $197(18.4)$ \\
Total & $239(12.0)^{*}$ & $759(33.4)$ & $998(23.4)$ \\
\hline
\end{tabular}

Data are $\mathrm{n}(\%)$. *: men compare with women $p<0.001$

Table 2: Prevalence of metabolic syndrome by gender and age group in untreated individuals. 
IDH (18.7\%) or ISH (23.2\%); women compared with men showed significantly higher presence of MS in all three hypertension subtypes; SDH was the most prevalent hypertension subtype (67.8\%) in people with MS; persons with only one MS component were the most common whatever hypertension subtypes.

Previous studies in China have shown that SDH was the most common hypertension subtype and participants with $\mathrm{SDH}$ were at the highest risk for any cardiovascular event $[11,12,14]$. The results of this current investigation reinforce the findings of previous study, which showed that SDH was the most prevalent hypertension subtype. In contrast, some studies reported that ISH was the most common hypertensive subtype and was associated with a higher risk of cardiovascular disease [15-17]. The discrepancies across studies were unclear; however, age distribution might be an important reason. Evidence from previous studies showed that the relative importance

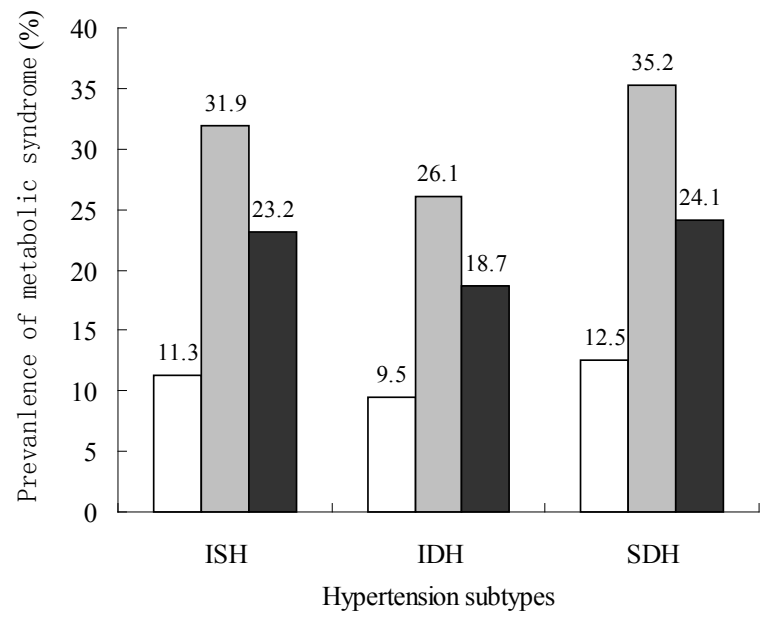

No shading, men; grey shading, women; black shading, combined men and women. ISH, isolated systolic hypertension; IDH, isolated diastolic hypertension; $\mathrm{SDH}$, systolic-diastolic hypertension. $p<0.05$, comparing prevalence of metabolic syndrome across all hypertensive subtypes; ${ }^{*} p$ $<0.001$, favoring higher prevalence of metabolic syndrome in women as compared with men.

Figure 1: Prevalence of the metabolic syndrome in untreated individuals.

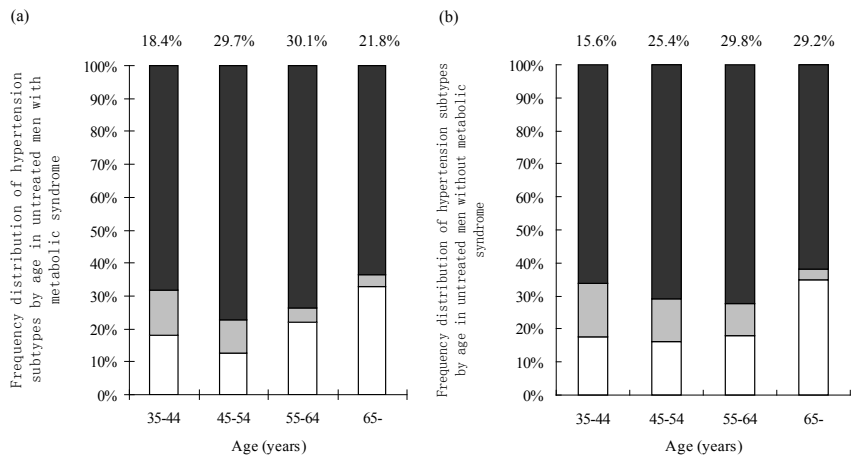

Figure 2: Frequency distribution of hypertension subtypes by age in untreated men with metabolic syndrome (a) and those without metabolic syndrome (b). Numbers at the tops of bars represent the overall percentage distribution of all subtypes of untreated hypertension in the age group. No shading, isolated systolic hypertension (SBP $\geq 140 \mathrm{mmHg}$ and DBP $<90$ $\mathrm{mmHg}$ ); grey shading, isolated diastolic hypertension (SBP $<140 \mathrm{mmHg}$ and $\mathrm{DBP} \geq 90 \mathrm{mmHg}$ ); black shading, systolic-diastolic hypertension (SBP $\geq 140$ $\mathrm{mmHg}$ and $\mathrm{DBP} \geq 90 \mathrm{mmHg}$ ).
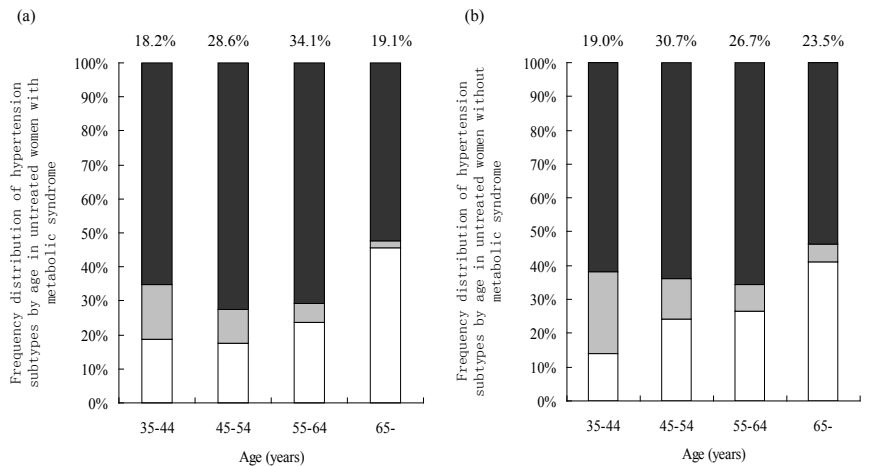

Figure 3: Frequency distribution of hypertension subtypes by age in untreated women with metabolic syndrome (a) and those without metabolic syndrome (b). Numbers at the tops of bars represent the overall percentage distribution of all subtypes of untreated hypertension in the age group. No shading, isolated systolic hypertension (SBP $\geq 140 \mathrm{mmHg}$ and DBP $<90$ $\mathrm{mmHg}$ ); grey shading, isolated diastolic hypertension (SBP $<140 \mathrm{mmHg}$ and $\mathrm{DBP} \geq 90 \mathrm{mmHg}$ ); black shading, systolic-diastolic hypertension (SBP $\geq 140$ $\mathrm{mmHg}$ and $\mathrm{DBP} \geq 90 \mathrm{mmHg}$ ).

of SBP decreased with decreasing age, further compared with SBP, DBP was a stronger predictor of coronary heart disease morbidity and mortality when subjects aged $\leq 45$ years [18]. Other study also found that ISH was the most common hypertension subtype in participants of age $\geq 50$ years. Conversely, for the younger hypertensive group, IDH was the most frequent form of hypertension and was comparable in prevalence with SDH [19].

The differences in proportion of hypertension subtype result in different prevalence of MS. In the current study, MS was more prevalent in patients with SDH rather than people with ISH or IDH. Different result was found by Franklin et al. that IDH subtype was associated with greatest likelihood of MS [20]. There was no sufficient explanation for these differences, the heterogeneity of the study populations may be an important reason and biological interactions between risk factors may be another important reason. As study pointed that age, daily cigarette smoking dose and the types of vitamins ingested was probably the most important risk factor for ISH, IDH and SDH, respectively. However, the other risk factors such as being age, family history of tumors, frequency of consumption of seafood, body weight, and total number of cigarettes were also associated with the different subtypes of hypertension [21].

In conclusion, the present study documented a high prevalence of SDH among rural hypertensive Chinese adults and showed significant association between hypertension subtype and MS, although differences were observed between studies. Since SDH was the most common form of hypertension and also the most prevalent hypertension subtype with MS, optimal reduction in cardiovascular risk frequently required concurrent treatment of both hypertension and other elements of MS in this rural hypertensive population.

Our study has several limitations. The major limitation is the crosssectional design, which cannot establish causal relations but can only generate hypotheses about the associations between hypertension subtype and MS of the participants. In this study, the sample contains only hypertensives, lacking normotensives for comparison. Other limitations include the possible misclassification of recall bias and confounding factors. 
Citation: Zhang X, Yao S, Sun Z, Zheng L, Xu C, et al. (2012) Metabolic Syndrome and Hypertension Subtypes among Untreated Hypertensive Rural Chinese. J Nutr Disorders Ther 2:111. doi:10.4172/2161-0509.1000111

\section{References}

1. Ford ES, Giles WH, Dietz WH (2002) Prevalence of the metabolic syndrome among US adults: findings from the third National Health and Nutrition Examination Survey. JAMA 287: 356-359.

2. Hu G, Qiao Q, Tuomilehto J, Balkau B, Borch-Johnsen K, et al. (2004) Prevalence of the metabolic syndrome and its relation to all-cause and cardiovascular mortality in nondiabetic European men and women. Arch Intern Med 164: 1066-1076.

3. Ford ES (2004) The metabolic syndrome and mortality from cardiovascular disease and all-causes: findings from the National Health and Nutrition Examination Survey II Mortality Study. Atherosclerosis 173: 309-314.

4. Marroquin OC, Kip KE, Kelley DE, Johnson BD, Shaw LJ, et al. (2004) Metabolic syndrome modifies the cardiovascular risk associated with angiographic coronary artery disease in women: a report from the Women's Ischemia Syndrome Evaluation. Circulation 109: 714-721.

5. Chen HJ, Bai CH, Yeh WT, Chiu HC, Pan WH (2006) Influence of metabolic syndrome and general obesity on the risk of ischemic stroke. Stroke 37:10601064.

6. Chien KL, Hsu HC, Sung FC, Su TC, Chen MF, et al. (2007) Metabolic syndrome as a risk factor for coronary heart disease and stroke: an 11-year prospective cohort in Taiwan community. Atherosclerosis 194: 214-221.

7. Thomas F, Bean K, Pannier B, Oppert JM, Guize L, et al. (2005) Cardiovascular mortality in overweight subjects. The key role of associated risk factors. Hypertension 46:654-659.

8. Khaw KT, Barrett-Connor E, Suarez L, Criqui MH (1984) Predictors of stroke associated mortality in the elderly. Stroke 15: $244-248$.

9. Petrovitch H, Curb JD, Bloom-Marcus E (1995) Isolated systolic hypertension and risk of stroke in Japanese-American men. Stroke 26: 25-29.

10. Pini R, Cavallini MC, Bencini F, Silvestrini G, Tonon E, et al. (2002) Cardiovascular remodeling is greater in isolated systolic hypertension than in diastolic hypertension in older adults: The Insufficienza Cardiaca negli Anzian Residenti (ICARE) a Dicomano Study. J Am Coll Cardiol 40: 1283-1289.

11. Fang $X H$, Zhang $X H$, Yang $Q D$, Dai $X Y$, Su FZ, et al. (2006) Subtype hypertension and risk of stroke in middle-aged and older Chinese: a 10-year follow-up study. Stroke 37: 38-43.

12. Kelly TN, Gu D, Chen J, Huang JF, Chen JC, et al. (2008) Hypertension subtype and risk of cardiovascular disease in Chinese adults. Circulation 118:1558-1566.

13. Alberti KG, Zimmet P, Shaw J, IDF Epidemiology Task Force Consensus Group (2005) The metabolic syndrome-a new worldwide definition. Lancet 366 1059-1062.

14. Strandberg TE, Salomaa VV, Vanhanen HT, Pitkala K, Miettinen TA (2002) Isolated diastolic hypertension, pulse pressure, and mean arterial pressure as predictors of mortality during a follow-up of up to 32 years. J Hypertens 20 : 399-404.

15. Nielsen WB, Lindenstrom E, Vestbo J, Jensen GB (1997) Is diastolic hypertension an independent risk factor for stroke in the presence of normal systolic blood pressure in the middle-aged and elderly? Am J Hypertens 10 634-639.

16. Petrovich H, Curb JD, Bloom-Marcus E (1995) Isolated systolic hypertension and risk of stroke in Japanese-American men. Stroke 26: 25-29.

17. Hozawa A, Ohkubo T, Nagai K, Kikuya M, Matsubara M, et al. (2000) Prognosis of isolated systolic and isolated diastolic hypertension as assessed by selfmeasurement of blood pressure at home: the Ohasama study. Arch Intern Med 160: 3301-3306

18. Antikainen R, Jousilahti P, Tuomilehto J (1998) Systolic blood pressure isolated systolic hypertension and risk of coronary heart disease, strokes, cardiovascular disease and all-cause mortality in the middle-aged population. J Hypertens 16: 577-583.

19. Franklin SS, Jacobs MJ, Wong ND, L'Italien GJ, Lapuerta P (2001) Predominance of isolated systolic hypertension among middle-aged and elderly US hypertensives. Analysis based on National Health and Nutrition Examination Survey (NHANES) III. Hypertension 37: 869-874.

20. Franklin SS, Barboza MG, Pio JR, Wong ND (2006) Blood pressure categories hypertensive subtypes, and the metabolic syndrome. J Hypertens 24: 2009 2016.

21. Wu H, Xu J, Zhuo L, Han L, Bao W, et al. (2009) Comparison of risk factors associated with hypertension subtypes by classification tree method in Tongshan County of Jiangsu Province, China. Am J Hypertens 22:1287-1294. 